\title{
Leptomeningeal Carcinomatosis in a Krukenberg Tumor Associated with Signet Ring Cell Gastric Cancer
}

\author{
Bilgin Bahadır BAŞGÖZ,' Birol YILDIZ, ${ }^{2}$ Adem AYDIN, ${ }^{1}$ \\ Gökhan ERDEM, ${ }^{2}$ Harun ASLAN, ${ }^{1}$ Kenan SAĞLAM, ${ }^{1}$ Fikret ARPACI ${ }^{2}$ \\ 'Department of Internal Medicine, Gülhane Training and Research Hospital, Ankara-Turkey \\ ${ }^{2}$ Department Medical Oncology, Gülhane Training and Research Hospital, Ankara-Turkey
}

\begin{abstract}
SUMMARY
Leptomeningeal carcinomatosis (LCM) is a rare complication of gastric cancer. It usually occurs late in advanced stage of disease and is sometimes misdiagnosed as toxicity of chemotherapeutic agents. Here we report a rare case of gastric cancer that developed LCM in follow-up. A 28-year-old woman with signet ring cell gastric cancer associated with Krukenberg tumor was admitted with persistent headache, nausea, vomiting, vertigo, and diplopia. Linear appearance of contrast enhancement in cerebellar fissures and around cranial nerves was seen in magnetic resonance imaging and cerebrospinal fluid was hypercellular with numerous carcinoma cells. LMC was confirmed and treated with intrathecal methotrexate and additional whole-brain irradiation. LCM is a rare complication, but occurs more often than expected and is often misdiagnosed. If patient who is being treated for gastric cancer presents with neurological symptoms, LCM should be kept in mind. Clinical improvement can be achieved with current treatment modalities, including radiotherapy, chemotherapy, or targeted molecules.
\end{abstract}

Keywords: Gastric cancer; Krukenberg tumor; leptomeningeal carcinomatosis; signet ring cell.

Copyright $\odot$ 2016, Turkish Society for Radiation Oncology

\section{Introduction}

Leptomeningeal carcinomatosis (LMC), also known as leptomeningeal metastases, is a clinically important and severe complication in patients with cancer and defined as diffuse spreading of malignant cells throughout the arachnoid membrane and the pia mater by propagation in the cerebrospinal fluid (CSF).[1]

It is associated with major neurologic symptoms and disability such as headache, nausea, vomiting, backache, radiculopathies, cranial nerve palsies, mental change and high mortality. [2,3] It can be observed in $3-8 \%$ of all cancer patients, and diagnosed in $1-5 \%$ of patients with solid tumors.[2,3] Although it may occur with virtually any malignancy, adenocarcinoma is the most common form of LMC and is most common- ly seen in patients with breast cancer, lung cancer- particularly small cell, also leukemia and lymphoma.[4,5] LMC is thought to be relatively rare in gastric cancer, with a frequency of $0.16-0.69 \%$ in all gastric cancer patients.[6,7]

Here we report a rare case of signet ring cell gastric cancer associated with Krukenberg tumor developed LMC in follow-up who was treated with concurrent radiotherapy and chemotherapy.

\section{Case Report}

A 28-year-old woman was admitted with persistent headache, nausea, vomiting, vertigo and diplopia. Her complains had lasted approximately for 30 days and became more apparent last week. 
In patient's medical history, it's learned that she was diagnosed as signet ring cell gastric cancer 22 months ago and total gastrectomy was performed. 6 cycles of 5-fluorouracil (5-FU)/folic acid and 45 Gy of radiotherapy was administered in follow.

14 months later from the diagnose date, $17 \mathrm{~cm}$ right ovarian mass detected in her routine follow-up and she had a surgery of right salpingo-oophorectomy. She was diagnosed as Krukenberg tumor and 6 cycles of docetaxel, cisplatin, and 5-FU (DCF) chemotherapy was planned but due to the side effects of the therapy she was discontinued her therapy after the first cycle.

In her physical examination her weight was $52 \mathrm{~kg}$ and height was $173 \mathrm{~cm}$. She was oriented to time, place and person. Her vital signs were as follows: a blood pressure of $110 / 70 \mathrm{mmHg}$, a pulse rate of 88 beats/min and a respiration rate of $17 \mathrm{breaths} / \mathrm{min}$. She had neck stiffness but doesn't have fever or skin rash. She had temporary spontaneous nystagmus and dysarthria. Psychomotor slowing was detected as a sign of an alteration on her mental status. Both lower extremities had paresis with preserved deep tendon reflexes. Plantar responses were flexor. Sensory examinations were normal. Hepatosplenomegaly was not noted.

In her laboratory tests no abnormality was detected except low hemoglobin levels of 9,6 g/dL (10.7-13.0 g/ $\mathrm{dL}$ ) and highly CA 19-9 levels, elevated from $156.6 \mathrm{U} /$ $\mathrm{ml}$ to $1901.6 \mathrm{U} / \mathrm{ml}(0-35 \mathrm{U} / \mathrm{ml})$ within 3 months.

Magnetic resonance imaging (MRI) study of brain with intravenous contrast was performed. Linear appearance of contrast enhancement was shown in cerebellar fissures and around cranial nerves.

A lumbar puncture and analysis of the cerebrospinal fluid (CSF) was performed. Gram stain and culture were negative. The results showed an elevated protein concentration with normal glucose content and hyper cellularity with many malignant cells.

Radiological diagnosis of LMC was confirmed through the results and the patient was treated with intrathecal administration of $15 \mathrm{mg}$ methotrexate, once a week, for 3 times. Additional whole-brain irradiation of $20 \mathrm{~Gy}$ in daily fractions was performed.

By the therapy she had dramatic relief from headaches, nausea, vomiting and diplopia. She became enable to feed orally and was discharged after had been planned DCF systemic chemotherapy of 6 cycles.

\section{Discussion}

LMC is very rare complication of gastric cancer, occurring in $0.16-0.69 \%$ of all gastric cancer patients.
$[6,7]$ However, recent studies showed that frequency of LCM is might be more often than known before. Emoto and colleagues [8] reported that $5.6 \%$ of patients with peritoneal metastasis of gastric cancer developed LCM during the course of therapy.

Breast cancer, melanoma and lung cancer are the most common solid tumors associated to LCM.[4,5] However, Lee et al.[6] reported that gastric cancer was most common solid tumor to metastasize to the meninges, accounting for $33 \%$, followed by lung cancer (31\%), and breast cancer (26\%).

The neurological symptoms of LMC are sometimes misdiagnosed as the toxicity of chemotherapeutic agents. We recommend scanning for LMC for patients with neurological symptoms that occurred during treatment. Recent studies suggest that the standard tool for imaging LMC is Gadolinium -enhanced MRI[9] and also cytology of the CSF is the gold standard for LMC cancer diagnosis, although false-negative results have often been reported.[10] That a combination of enhanced MRI and CSF cytology be used for accurate diagnosis of LMC.[10] Although MRI is generally regarded as superior to CT for the diagnosis of LMD, factors such as cost, availability and patient convenience often dictate that contrast-enhanced CT scans are the first studies ordered for the patient with suspected LMD, especially in the acute clinical setting when hydrocephalus should be excluded prior to lumbar puncture.[11]

The prognosis of LMD is unpromising without treatment, with an average survival of 6 weeks following diagnosis.[12] With treatment, the average survival is 4 to 6 months [4] with survival tending to be longer in patients with breast cancer or hematologic malignancies.

The goals of treatment include prolonging survival, improve neurologic function if not possible palliating symptoms. Intrathecal (IT) chemotherapy using MTX, cytosine arabinoside (Ara-C), and thiotepa is the mainstay of the treatment of LMC,[11] though the efficacy and superiority to systemic chemotherapy of this regimen is still unclear. A recent study shows that combination of MTX, Ara-C and hydrocortisone found more effective than the administration of MTX alone in LCM associated with solid tumors.[13] A standard induction therapy $10 \mathrm{mg}$ twice a week for four weeks. If there is no response, additional four-week induction therapy or an alternative approach may be considered.[14] Recently systemic administration of targeted therapies in selected patients with LMC resulted in clinical benefit as example erlotinib and alectinib in lung cancer.[15,16] However large-scale clinical studies are required for 
standardizing intrathecal chemotherapy. On the other hand, Radiation therapy (RT) appears to be more effective at relieving symptoms than does (IT) chemotherapy for symptoms caused by localized leptomeningeal metastases. Also whole-brain radiation is often performed for LMC patients to palliate their symptoms, decrease bulky disease, and correct CSF flow abnormalities. [8] 30-36 Gy in 3 Gy daily fractions is recommended by several studies.[17,18] CSF drainage is also performed to relieve the symptoms of elevated intracranial pressure.[19] Corticosteroids is rarely effective in neurological deficits but can improve headaches better than analgesics. Furthermore, anticonvulsants should not be used as prophylactic and reserved for patients with seizures. Emoto and colleagues[8] reported that in patients with poor prognostic factors such as poor performance status or MRI-proven LMC, palliative therapy may be the most suitable treatment strategy. In addition, the clinical efficacy of IT MTX in conjunction with RT is illustrated by several study.[20,21]

In summary, LCM is a rare complication of gastric cancer but recent studies imply that it occurs more than expected but usually might be misdiagnosed. Cytology of the CSF is the gold standard although to avoid from false-negative results, combination of enhanced MRI and CSF cytology be used for accurate diagnosis. Prognosis is poor and worsened if not treated. But, clinical studies are required for standardizing therapy. In conclusion, if the patient who treated for the gastric cancer presenting with neurological symptoms, should be excluded for LCM by clinician.

\section{Disclosure Statement}

The authors declare no conflicts of interest.

\section{References}

1. Braeuninger S, Mawrin C, Malfertheiner P, Schildhaus HU, Seiler C, Dietzmann K, et al. Gastric adenocarcinoma with leptomeningeal carcinomatosis as the presenting manifestation: an autopsy case report. Eur J Gastroenterol Hepatol 2005;17(5):577-9. Crossrel

2. Posner JB. Leptomeningeal metastases. In: Posner JB, editor. Neurologic complications of cancer. Philadelphia, PA: F. A. Davis Company; 1995. p. 143-71.

3. DeAngelis LM. Current diagnosis and treatment of leptomeningeal metastasis. J Neurooncol 1998;38(23):245-52. Crossree

4. Grossman SA, Krabak MJ. Leptomeningeal carcinomatosis. Cancer Treat Rev 1999;25(2):103-19. Crossret
5. Wasserstrom WR, Glass JP, Posner JB. Diagnosis and treatment of leptomeningeal metastases from solid tumors: experience with 90 patients. Cancer 1982;49(4):759-72. Crossret

6. Lee JL, Kang YK, Kim TW, Chang HM, Lee GW, Ryu $\mathrm{MH}$, et al. Leptomeningeal carcinomatosis in gastric cancer. J Neurooncol 2004;66:167-74. Crossre

7. Raj KP, Sanati H, Mehta RS, Zell JA. Need for a new treatment strategy: leptomeningeal carcinomatosis from gastric cancer. Anticancer Drugs 2009;20(4):3014. Crossre

8. Emoto $\mathrm{S}$, Ishigami $\mathrm{H}$, Yamaguchi $\mathrm{H}$, Yamashita $\mathrm{H}$, Kaisaki S, Kitayama J. Frequent development of leptomeningeal carcinomatosis in patients with peritoneal dissemination of gastric cancer. Gastric Cancer 2011;14(4):390-5. Crossree

9. Clarke JL, Perez HR, Jacks LM, Panageas KS, Deangelis LM. Leptomeningeal metastases in the MRI era. Neurology 2010;74(18):1449-54. Crossre

10. Groves MD. New strategies in the management of leptomeningeal metastases. Arch Neurol 2010;67(3):30512. Crossree

11. Lisenko Y, Kumar AJ, Yao J, Ajani J, Ho L. Leptomeningeal carcinomatosis originating from gastric cancer: report of eight cases and review of the literature. Am J Clin Oncol 2003;26(2):165-70. Crossree

12. Fisher MA, Weiss RB. Carcinomatous meningitis in gastrointestinal malignancies. South Med J 1979;72(8):930-2. Crossret

13. Kim DY, Lee KW, Yun T, Park SR, Jung JY, Kim DW, et al. Comparison of intrathecal chemotherapy for leptomeningeal carcinomatosis of a solid tumor: methotrexate alone versus methotrexate in combination with cytosine arabinoside and hydrocortisone. Jpn J Clin Oncol 2003;33(12):608-12. Crossrel

14. Siegal T, Lossos A, Pfeffer MR. Leptomeningeal metastases: analysis of 31 patients with sustained off-therapy response following combined-modality therapy. Neurology 1994;44(8):1463-9. Crossre

15. Dhruva N, Socinski MA. Carcinomatous meningitis in non-small-cell lung cancer: response to high-dose erlotinib. J Clin Oncol 2009;27(22):e31-2. Crossre

16. Gadgeel SM, Gandhi L, Riely GJ, Chiappori AA, West HL, Azada MC, et al. Safety and activity of alectinib against systemic disease and brain metastases in patients with crizotinib-resistant ALK-rearranged nonsmall-cell lung cancer (AF-002JG): results from the dose-finding portion of a phase $1 / 2$ study. Lancet Oncol 2014;15(10):1119-28. Crossree

17. Yuan Y, Tan C, Li M, Shen H, Fang X, Hu Y, et al. Activity of pemetrexed and high-dose gefitinib in an EGFR-mutated lung adenocarcinoma with brain and 
leptomeningeal metastasis after response to gefitinib. World J Surg Oncol 2012;10:235. Crossree

18. Nagano T, Kotani Y, Kobayashi K, Hatakeyama Y, Hori $\mathrm{S}$, Kasai D, et al. Long-term outcome after multidisciplinary approach for leptomeningeal carcinomatosis in a non-small cell lung cancer patient with poor performance status. Intern Med 2011;50(24):3019-22.

19. Omuro AM, Lallana EC, Bilsky MH, DeAngelis LM. Ventriculoperitoneal shunt in patients with leptomen- ingeal metastasis. Neurology 2005;64:1625-7. Crossre

20. Pfeffer MR, Wygoda M, Siegal T. Leptomeningeal metastases--treatment results in 98 consecutive patients. Isr J Med Sci 1988;24(9-10):611-8.

21. Sause WT, Crowley J, Eyre HJ, Rivkin SE, Pugh RP, Quagliana JM, et al. Whole brain irradiation and intrathecal methotrexate in the treatment of solid tumor leptomeningeal metastases--a Southwest Oncology Group study. J Neurooncol 1988;6(2):107-12. Crossree 